\title{
Small-world or Scale-Free Phenomena in Internet: What Implications for the Next-generation Networks?
}

\author{
Zouhaïer M'Chirgui \\ Euromed Management, LAREQUAD, Department of Information \& Finance \\ Domaine de Luminy, BP 921- 13288 Marseille cedex 9, France \\ E-mail: Zouhaier.MChirgui@euromed-management.com
}

$\begin{array}{ll}\text { Received: November 24, } 2011 & \text { Accepted: February 10, } 2012 \quad \text { Published: March 1, } 2012 \\ \text { doi:10.5539/res.v4n1p85 } & \text { URL: http://dx.doi.org/10.5539/res.v4n1p85 }\end{array}$

I am very gratefully to Thierry Pénard for his helpful suggestions and insights. This work was partially supported by the ACTRICE project.

\begin{abstract}
The paper examines the large-scale topological structure of the Internet in order to see whether the structure exhibits some features that lead Internet Services Providers to go aside further collaboration for the deployment of next-generation networks. The results indicate the existence of positive signs or early stages of possible move towards more cooperative relationships, mainly among backbones. These findings have implications for the next-generation networks policy and strategy, particularly the move towards strategic alliances after the recent phase of mergers and acquisitions.
\end{abstract}

Keywords: Next-generation networks, Internet, Alliances, Small-world, Scale-free, Internet services providers

\section{Introduction}

The debate in the literature about the next-generation networks rotates around two major issues relating to the evolution of economic business models and of regulations (see for example (Frieden, 2003, 2007). Whether the debate on regulation seems of most concern in the United States, while European institutions and Japanese government are just beginning to focus on (Cave et al., 2009; Wallsten and Hausladen, 2009, Cave 2010; Ruhle and Lundborg, 2010), it is important to consider the impact of any changes on the future of the industry. Further, the debate emphasizes two opposite views, which could be dealing with the 'Net Neutrality' controversy or neutrality restrictions. On the one side, some are against Net neutrality. They argue that Net neutrality rules would reduce incentives to upgrade networks and launch next generation network services. They underline that in the absence of Net neutrality pricing would be more flexible and Quality of Service (QoS) better achieved (Felten, 2006; Lessig, 2006; Hahn and Wallsten, 2006; Clarke, 2009). This argument is supported by Internet broadband providers AT\&T, Verizon and a number of TV companies. On the other side, others are against discrimination and support Net neutrality because of its primary importance to the preservation of current freedom (Economides and Tåg, 2007; Caves et al., 2009). They believe that broadband providers will use their market power to block the applications they do not favour and will also discriminate between contents providers (i.e. websites, services, protocols). This argument is supported by large web content companies such as Google, Yahoo or MSN.

Going along these debates, several questions could be raised. Who should bear the cost of the new-generation infrastructure? How can Quality of Service be achieved efficiently? How can cooperation and trust be strengthened between operators?

To answer these questions we need firstly to start to study the topological structure of ISPs' relationships. In other words, to see whether the current structure of interconnection network between ISPs exhibit some features that enable to go for further collaboration, and at which level of the structure this is more likely to occur. Though some studies have examined the topological structure of Internet (Amaral et al., 2000; Barabási, 2002; Vásquez 
et al.,2002; Yan and Assimakopoulos, 2009), to the best of my knowledge, none has addressed the strategic implications.

We argue our approach or method by two points. First, ISPs are already bound by a preliminary agreement of collaboration through peering agreements. Indeed, to achieve universal connectivity and exchange traffic Internet providers have to settle interconnection agreements. Interconnection agreements are of two sorts: peering and transit. Whether transit agreement involves a fees payment by customer to provider, a peering agreement is a bilateral peer-to-peer relationship where Internet providers do not charge each other for terminating traffic. It involves the exchange traffic for free between them. Therefore, the peering agreement could be considered as a preliminary agreement of collaboration between two Internet providers. Second, some recent studies have shown that asymmetric effect was one major factor that has a negative and significant impact on tying a peering agreement (Carter and Wright, 2003; Weiss and Shin, 2004; Badasyan and Chakrabarti, 2008; Shrimali and Kumar, 2008; Jahn and Prüfer, 2008; Lippert and Spagnalo, 2008). Internet providers prefer tie peering with providers of similar size, otherwise transit agreements. One key interpretation that may be emphasized here is that Internet providers who are willing to tie peering agreements with other providers of similar size, could be also ready and willing to tie alliances with this same actors. So, peering can be considered as an incremental agreement to move toward more deeply relationship as strategic alliance (M'Chirgui and Pénard, 2008). So, it is prominent firstly to look whether the current interconnection structure amongst ISPs exhibits some features that can be interpreted as positive signs to go even further in their relationships.

\section{Theoretical Background}

Recent years studies on network topology have grown rapidly, particularly, in order to understand how topology affects both capacity and robustness of networks, as well as the efficiency of communication. For example, recent studies on economic activities network have indicated that the topological structure of inter-firm network influences the rate of diffusion as well as the efficiency of knowledge, competencies and resources exchange (Powell et al., 1996; Amaral et al., 2000; Kogut and Walker, 2001; Barabási, 2002; Vásquez et al., 2002; Baum et al., 2004; Schintler et al., 2005; Uzzi and Spiro, 2005; Goyal et al., 2006; Schilling and Pelhps, 2007).

Roughly speaking, mainly four network models designed to mimic real-world networks are usually found in the network literature and promoted by empirical studies: the regular network model, the random network model by Erdös and Rényi (1959), the small-world network model by Watts and Strogatz (1998) and the scale-free network model by Barabási and Albert (1999). Through these models researchers aim to understand the network formation process as well as interaction structure between actors.

Topological features such as degree distribution, clustering, and shortest path were three major concepts or pattern of connections used to define each kind of these network models, whether they share the same number of nodes $(n)$ and the same number of links $n k / 2$, ( $k$ number of ties). Short average path length, $L$, indicates that the distance between any two nodes on the network is short; they can be reached in a few number of steps between two nodes. The clustering coefficient, $C$, provides measure of how well locally interconnected are the neighbours of any node. The maximum value of the clustering coefficient $C$ is 1 ; corresponding to a fully connected network. For random networks, which are constructed by connecting nodes at random with a fixed probability $p$, the clustering coefficient decreases with the network size $n$ as $C=k / n$. on the contrary, it remains constant for regular lattices. While, the path length is a measure of the global structure of a network, because information about the topology of the entire network is required to determine the shortest path length between any two nodes, the clustering coefficient is in contrast a measure of local network structure. Lastly, the frequency distributions of node density, called degrees, often follow power laws. A power law distribution is a statistical distribution in which one variable is proportional to a power of the other. When plotted on a $\log / \log$ scale, individual points are distributed about a straight line. This means that there are a small number of nodes (the "hubs" (Note 1)) which have many neighbours and a large number of nodes that have only a few neighbours.

Regular network (Fig. 1(a)) is characterized by an identical local structure reproduction over the network. Each of the $n$ individuals may interact (be connected) only with the $k$ most immediate neighbours. In contrast, Random network (Fig. 1(b)), is a network in which links between individuals are arranged randomly, so that each pair has an equal probability to become connected. By rewiring each of the $n k / 2$ relations of the local network exactly once, each agent is now connected with an average of $k$ other individuals which could be located anywhere on the network. While the local network is unique once $n$ and $k$ are specified, random networks can take a variety of shapes.

Small-world network (Fig. 1(c)) has the properties of both regular and random networks. To obtain a small-world network, we start with a regular network and randomly rewire each of the $n k / 2$ connections of the local network 
with a very small probability. In this way, most of the agents are still connected to their closest neighbours, but now a very small number of new links can directly connect two agents that are far apart on the network. According to Watts and Strogatz (1998), random networks have short path lengths and exhibit little clustering, while small-world networks have a high clustering coefficient and a low average path length. These two characteristics are now considered as two basic statistical properties to detect small-world networks in empirical analysis. Thus, for a network to be a small-world, the ratio of the random and actual average path lengths should be approximately 1; and the small-world coefficient, $S W$, should be greater than 1 . The small-world coefficient is the ratio of the clustering coefficient of a network to that of a random network.

Scale-free network (Fig. 1(d)) emerges in the context of growing network in which new nodes connect preferentially to the more highly connected nodes in the network. Thus, the main feature of scale-free networks is that each node has a statistically significant probability of having a very high degree compared with the average degree $(k)$ of the network. These highly connected nodes dominate the topology of the network by forming hubs, which is not the feature of random or small-world networks. The scale-free model explains power law degree distributions however the small world model explains clustering (Albert and Barabási, 2002, p.49). The power-law fit implies that the network has no 'typical' node, in the sense that a Gaussian distribution would have a mean node, and the distribution is scale-invariant (networks are free of characteristic of scale; that's why it is called scale-free) (Barabási and Albert, 1999). Furthermore, the identification of either small-world or scale free topologies implies particular dynamic properties of the network e.g. stability (Li and Chen, 2003). However, scale-free networks have also, as for small-world networks, clustering coefficient much larger than random networks and their diameter increase logarithmically with the number of nodes, certain consider scale-free networks as small-world networks (Amaral et al., 2000).

$<$ Insert Figure 1 about here $>$

\section{Data and Sample}

The primary source of data used in this study was ISPs' relationships developed from BGP (Border Gateway Protocol) tables and provided by Orange. Data are monthly. They cover a period of eighteen months throughout the years 2005 and 2006. For each month, we then sampled data that included all ISPs of the Internet.

For our empirical research we focused mainly on the top hundred Internet providers that were active in this period. The sample includes all interconnection agreements established by the top hundred domains (to see how the rank is computed see Xia and Gao, 2004). The unit of analysis is the dyad, which represents two ISPs.

The sample choice is justified by the fact that Internet is characterized by a network hierarchical structure. Indeed, the economic relationships between the Internet providers are characterized by a three-tier structure. Tier 1 is composed of the global backbone transit networks (or Internet Backbone Providers - IBP). Tier 2 includes the national wholesale transit networks (or Internet Service Providers - ISP), but also some major content providers. Tier 3 gathers the local retail access ISPs (also known as Internet Access Providers - IAP) and the majority of content providers. That's why it is important to study the topological structure at each level and at the top level in particular as it shapes the heart of the Internet. Table 1 shows the distribution of ISPs according to their hierarchical level (Tier 1, 2, or 3) for three times over the period of analysis (18 months).

$<$ Insert Table 1 about here $>$

\section{Analysis and Results}

In the following, we examine the large-scale topology structure of the Internet. Mainly two network models will be examined. We start to examine small-world characteristics then scale-free properties. The small-world analysis would explain clustering and the scale-free analysis would explain the power law degree distribution. The small-world analysis deals with the study of the global structure however the scale-free analysis enables to investigate the sources of the global structure and consequently to understand the position of Internet providers.

\subsection{Small-world analysis}

To test whether the network of interconnection agreements in Internet could be classified as having small-world topology, we calculate the two basic statistical properties of networks: the clustering coefficient, $C(C=$ number of links between neighbours / total number of links), and the characteristic path-length, $L$, and compare them to a randomly connected network of the same size, $n$, and nodes degree distribution, $k$, respectively. A small-world network is characterized by a high degree of local clustering or high values of $C$, indicating that the network is strong densely connected, and a short average minimum path or low values of $\mathrm{L}$, indicating that knowledge and resources can move from one firm to another in a relatively small number of intermediaries. Empirically, in order to determine characteristic of the small-world network topology, we compare the values of these two 
statistical properties $(C$ and $L)$ for the actual network in question, with their values for a random network (respectively noted, $C_{R}$ and $L_{R}$ ). Watts (2003) defines a Small-world graph as any graph with $n$ vertices and average vertex degree $k$ that exhibits $L \approx L_{R}(n, k) \sim \ln (n) / \ln (k)$ and $C \gg>C_{R} \sim k / n$ for $n \gg>>>\ln (n)>1$. Further, we calculate the small-world coefficient, $S W$, that should be greater than 1 (Davis et al., 2003). The small-world coefficient is the ratio of the clustering coefficient of a network to that of a random network. Following Kogut and Walker (2001) and Baum et al., (2004), the SW is defined as follows: $S W=\left(C / C_{R}\right) /\left(L / L_{R}\right)$

Table 2 summarizes the evolution of small-world properties of the Internet network taking into account all agreements covered by the database. The table gives information about the numbers of ISPs involved in interconnection agreements for each month $(n)$ over the period of analysis, the average number of links by ISPs $(k)$ or the average degree (Av. Degree), the diameter (Dia), the average path length for the actual networks $(L)$ and random networks $\left(L_{R}\right)$, the clustering coefficient for the actual networks $(C)$ and random networks $\left(C_{R}\right)$, and ratios comparing the values both for path length and clustering coefficient. Finally, the last column of the table gives values for the small-world statistic $(\mathrm{SW})$.

$<$ Insert Table 2 about here $>$

It can be derived from the Table 2, that the Internet interconnection agreements network strikingly exhibits "small-word" effect. The statistical properties have a threshold, which satisfy the definition of a small-world network topology. The ratios of actual and random average path lengths for all months are approximately $1(L \approx$ $L_{R}(n, k)$ ); and the small world coefficients are greater than 1 (it means that $C>>C_{R}$ ).

Now in order to focus deeply on the evolution of interconnection agreements we mainly focus on the network structure at Tier 1 and Tier 2. We have built a new database which contains interconnections agreements tied only by the top hundred ISPs. Then, from this database we have made two others databases. One presents agreements shaped by Tier 1 . The other presents agreements shaped by Tier 2 .

The choice is motivated by the fact that backbones draw the heart of the Internet, allowing the networks that make up the Internet to exchange traffic and all other ISPs (non tier 1) can be broadly categorized as Tier 2 in the peering context. This situation shows on the one hand the complexity of market structure where local and global internet providers cooperate and compete to offer end-users a wide range of services and on the other hand the asymmetry that exist between internet providers inducing hierarchical pricing. Consequently, it is important to examine separately the network structure at each level of tiers.

Table 3 summarizes the evolution of small-world properties of the three types of Internet provider networks (Top 100 ISPs network, Tier1 network and Tier2 network) through three snapshots of six-month periods. Table 3 shows that the three networks clearly exhibit "small-word" characteristics. The statistical properties have a threshold, which satisfy the definition of a small-world network topology as defined previously. Interestingly, the degree of small-worldless differs for the three networks. Tier 1 network exhibits the more prominent characteristics of small world effect.

$<$ Insert Table 3 about here $>$

One interpretation of this result is that, on the one hand, there is a strong cohesion and more stability at the top level and on the other hand, the network is likely to be weakly connected and more unstable at the lower level. The originality of this topological analysis compared to the previous works was to highlight the dual structure of the Internet.

\subsection{Scale-free analysis}

According to Albert and Barabási, (2002) Scale-free properties evolve in large complex networks through self-organizing processes and satisfy three elements. Initial condition, the network consists of a small number of nodes and no links. Then, the network should growth. Finally, the existence of preferential attachment; it means that the probability $P$ that the new node will be connected to node $i$ depends linearly on the degree $k_{i}$. New nodes are more probably to link up with existing nodes that have large centrality degrees than to nodes with relatively lower degrees. Consequently, highly connected nodes become more connected over time. These highly connected nodes dominate the topology of the network by forming hubs, which is not the feature of random or small-world networks. Therefore, the centrality of these nodes functions as an attractive element for new nodes to join the network. To check the third condition we must examine the degree distribution or the frequency distributions of node density. A preferential attachment occurs with the presence of power law distribution. A power law distribution is a statistical distribution in which one variable is proportional to a power of the other. When plotted on a $\log / \log$ scale, individual points are distributed about a straight line.

$<$ Insert Figure 2 about here $>$ 
Figures $2 \mathrm{a}, 2 \mathrm{~b}$ and $2 \mathrm{c}$ exhibit a $\log -\log$ representation of the cumulative degree distribution for the three interconnection agreements networks in order to assess whether the distributions follow a power law (in which case one would obtain a linear relationship in log-log scale). The figures indicate an incomplete power-law relation, since the individual points of the degree distribution are not distributed along a straight line when plotted in log-log scale. This result is of importance, as it partly diverges from the 'physicists' explanation of the dynamic network formation à la Barabasi (2002). Given that our empirical cases lie in an intermediate position between a Gaussian and a power-law distribution, we concluded that interconnections networks are not totally cannibalized by a few Internet providers, in spite of the crucial role played by the main actors. Moreover, the figure 2(b) corresponding to Tier 1 is more likely to be close to scale-free distribution.

\section{Implications and Conclusion}

In this paper we have examined the large-scale topology structure of the Internet in order to see whether ISPs are ready to move towards more cooperative relationships to co-invest in the next-generation network. Interestingly, the results of both analysis (scale-free and small-world) indicate that Tier 1 exhibits strong cohesion, more stability and likely to functions as an attractive element for Internet providers. These facilitate the development of trust and reciprocity norms that can increase individual's willingness to exchange information (Bouty, 2000). Indeed, backbones, or Tier 1, shape the heart of the Internet. They guarantee universal connectivity and play a central role in the quality of connectivity. Yet, they act as an essential intermediaries through provider-customer agreements that they sign with ISPs at lower level (these agreements generate a stream of income from the end-customer to these backbones). The introduction of new business models and new services cannot be done without these central and dominant players. Therefore, the adoption of new organizational forms that involve more trust and stability among actors is more likely to occur at (or to concern) the Tier 1 level.

Moreover, the shift towards a new Internet interconnection generation (QoS interconnection) is becoming a critical issue for the backbones and ISPs that wish to achieve end-to-end QoS, (Hwang and Weiss, 2007). Investments in next-generation networks are very important. Further, a better routing mechanism with better QoS and traffic optimization requires a high level of coordination and information exchange among operators and consequently a high degree of trust and stability in their relationships. Therefore, it appears likely impossible to build the next-generation networks in a non cooperative way. Thus, forming alliances could be an optimal issue to overcome this problem. It is a cooperative way to produce more stability and trust among ISPs and achieve better network service performance (Rousseau et al., 1998, Parkhe, 1998; Kauser and Shaw, 2004; Schumacher, 2006). Indeed, the study of the structure of interconnection agreements network has shown the existence of positive signs or early stages of possible move towards more cooperative relationships, mainly among backbones.

Alliances may be the next major organizational mode for the Internet after the recent phase of mergers and acquisitions (Buccirossi et al., 2005, D’Ignazio and Giovannetti, 2006). Alliances between Internet providers will be a major means of developing a common and interoperable QoS interconnection infrastructure through which operators supply differentiated services involving differentiated pricing (Economides and Tåg, 2007).

\section{References}

Albert, R. \& Barabási, A. L. (2002). Statistical mechanics of complex networks. Review of Modern Physics, 74, 47-97. http://dx.doi.org/10.1103/RevModPhys.74.47

Amaral, L. A. N., Scala, A., Barthélémy, M. \& Stanley, H. E. (2000). Classes of small-world networks' PNAS, 97(21), 11149-11152. http://dx.doi.org/10.1073/pnas.200327197

Badasyan, N. \& Chakrabarti, S. (2008). A simple game-theoretic analysis of peering and transit contracting among Internet service providers. Telecommunications Policy, 32(1), 4-18. http://dx.doi.org/10.1016/j.telpol.200 7.11 .002

Barabási, A. L. \& Albert, R. (1999). Emergence of scaling in random network, Science, 286, 509-512. http://dx.doi.org/10.1126/science.286.5439.509

Barabási, A. L. (2002). Linked: The New Science of Networks. Cambridge, MA: Perseus Publishing.

Baum, J., Shipilov, A. \& Rowley, T. (2003). Where do Small-Worlds come from? Industrial and Corporate Change, 12, 697-725. http://dx.doi.org/10.1093/icc/12.4.697

Baum, J., Shipilov, A. \& Rowley, T. (2004). The 'small-world' of Canadian capital markets: Statistical mechanisms of investment bank syndicate networks, 1952-1989, Canadian Journal of Administrative Sciences, 21, 307-325. http://dx.doi.org/10.1111/j.1936-4490.2004.tb00347.x 
Bouty, I. (2000). Interpresonal and interaction influences on informal resource exchanges between R\&D researchers across organizational boundaries. Academy of Management Journal, 43, 50-66. http://dx.doi.org/10. $2307 / 1556385$

Buccirossi, P., Ferrari, B. L. \& Siciliani, P. (2005). Competition in the Internet Backbone Market, World Competition, 28(2), 235-254.

Carter, M. \& Wright, J. (2003). Asymmetric Network Interconnection, Review of Industrial Organization, 22, 27-46. http://dx.doi.org/10.1023/A:1022199902266

Cave ,M. (2010). Snakes and ladders: Unbundling in a next generation world. Telecommunications Policy, 34, 80-85. http://dx.doi.org/10.1016/j.telpol.2009.11.006

Cave M., Collins R., van Eijk N., Larouche P., Prosperetti, L., de Streel A., \& Valletti, T. (2009). Statement by European academics on the inappropriateness of imposing increased internet regulation in the EU. [Online] Available: http://ssrn.com/abstract=1329926

Clarke, R. (2009). Costs of Neutral/Unmanaged IP Networks? Review of Network Economics, 8(1): 61-89. http://dx.doi.org/10.2202/1446-9022.1170

D’Ignazio, A. \& Giovannetti, E. (2006). Antitrust Analysis for the Internet Upstream Market: A Border Gateway Protocol Approach. Journal of Competition Law and Economics, 2(1).

Davis, G. F., Yoo, M. \& Baker, W. E. (2003). The small world of the corporate elite, 1982-2001. Strategic Organization, 1, 301-326. http://dx.doi.org/10.1177/14761270030013002

Economides, N. \& Tag, J. (2007). Net neutrality on the Internet: a two sided market analysis. working paper 07-04, Net Institute.

Erdos, P. \& Renyi, A. (1959). On random graphs. Pulicationes Mathematicae, 6, 290-297.

Felten, E. W. (2006). Nuts and Bolts of Network Neutrality, Center for Information Technology Policy, Department of Computer Science, and Woodrow Wilson School of Public and International Affairs Princeton University.

Frieden, R. (2003). Regulation for Internet-mediated communication and commerce. in Gary Madden (ed.). Emerging Telecommunications Networks. Volume II of The International Handbook on Telecommunications Economics, Cheltenham: Edward Elgar, 107-128.

Frieden, R. (2007). Network Neutrality or Bias?--Handicapping the Odds for a Tiered and Branded Internet. 29 Hastings Communications and Entertainment Law Journal, 2, 171-216.

Goyal, S., Van De Leij, M. \& Moraga-Gonzalez, J. L. (2006). Economics: An emerging 'small-world'? Journal of Political Economy, 114, 403-412. http://dx.doi.org/10.1086/500990

Hahn, R. \& Wallsten, S. (2006). The Economics of Net Neutrality. The Economists' Voice, 3/6.

Jahn, E. \& Prüfer, J. (2008). Interconnection and competition among asymmetric networks in the Internet backbone market, Information Economics and Policy, 20(3), 243-256. http://dx.doi.org/10.1016/j.infoecopol.200 8.03.002

Kogut, B. \& Walker, G. (2001). The 'Small-World' of Germany and the durability of national networks. American Sociological Review, 66, 317-335. http://dx.doi.org/10.2307/3088882

Lessig, L. (2006). Testimony of Larry Lessig, Hearing on Network Neutrality. Senate Committee on Commerce, Science and Transportation.

Li, C. \& Chen, G. (2003). Stability of a neural network model with small-world connections. Physical Review E., $68,052901 / 1-052901 / 4$.

Lippert, S. \& Spagnolo, G. (2008). Internet Peering as a Network of Relations. Telecommunications Policy, 32(1), 33-49. http://dx.doi.org/10.1016/j.telpol.2007.11.004

M'Chirgui, Z. \& Pénard, T., (2008). How to provide Quality of Service guarantees in the Internet? The lessons from the airline and card-based payment sectors. Proceeding of the 4th IEEE International Conference on Management of Innovation and Technology, IEEE, ISBN: 978-1-4244-2330-9, pp.923-927.

Powell, W., Koput, K. \& Smith-Doerr, L. (1996). Interorganizational collaboration and the locus of invention: networks of learning in biotechnology. Administrative Science Quarterly, 41, 116-145. http://dx.doi.org/10.2307 $/ 2393988$ 
Ruhle, E. O. \& Lundborg, M. (2010). EU policy for next generation access - an innovative or incremental step forward? Telecommunications Policy, 34, 36-44. http://dx.doi.org/10.1016/j.telpol.2009.11.007

Schilling, M. \& Phelps, C. (2007). Interfirm collaboration networks: The impact of large scale network structure on firm innovation. Management Science, 53(7), 1113-1126. http://dx.doi.org/10.1287/mnsc.1060.0624

Schintler, A., Gorman, S. P., Reggiani, A., Patuelli, R., Gillespie, A., Nijkamp, P. and Rutherford, J. (2005) Complex network phenomena in telecommunication systems. Networks and Spatial Economics, 5, 351-370. http: //dx.doi.org/10.1007/s11067-005-6208-z

Shrimali, G. \& Kumar, S. (2008). Bill-and-keep peering. Telecommunications Policy, 32(1), 40-53. http://dx.doi. org/10.1016/j.telpol.2007.11.007

Uzzi, B. \& Spiro, J. (2005). Collaboration and Creativity: The Small World Problem. American Journal of Sociology, 111(2), 447-504. http://dx.doi.org/10.1086/432782

Vásquez, A., Pastor-Satorras, R. \& Vespignani, A. (2002). Large scale topological and dynamical properties of the Internet. Physical Review E, 65, 066130. http://dx.doi.org/10.1103/PhysRevE.65.066130

Wallsten, S. \& Hausladen, S. (2009). Net Neutrality, Unbundling, and their Effects on International Investment in Next-Generation Networks. Review of Network Economics, 8(1), 90-112. http://dx.doi.org/10.2202/1446-9022 .1171

Watts, D. J. \& Strogatz, S. H. (1998). Collective dynamics of “small-world” networks. Nature, 393, 440-2. http://dx.doi.org/10.1038/30918

Weiss, M. B. \& Shin, S. J. (2004). Internet interconnection economic model and its analysis: Peering and settlement. Netnomics, 6, 43-57. http://dx.doi.org/10.1023/B:NETN.0000023380.38300.25

Xia, J. \& Gao, L. (2004). On the Evaluation of AS Relationship Inferences. IEEE Globecom.

Yan, J. \& Assimakopoulos, D. (2009). The small-world and scale-free structure of an internet technological community. International Journal of Information Technology and Management, 8, 33-48.

Note:

Hub is a node with a disproportionately high number of connections to other nodes. In scale-free networks, hubs may have node degrees several orders of magnitude higher than the degrees of other nodes. Hub is a node with a disproportionately high number of connections to other nodes. In scale-free networks, hubs may have node degrees several orders of magnitude higher than the degrees of other nodes.

Table 1. The distribution of ISPS

\begin{tabular}{|l|l|l|l|}
\hline & $2005 \_07$ & $2006 \_01$ & $2006 \_07$ \\
\hline Tier 1 & 13 & 12 & 19 \\
\hline Tier 2 & 71 & 73 & 69 \\
\hline Tier 3 & 16 & 15 & 12 \\
\hline Total & 100 & 100 & 100 \\
\hline
\end{tabular}


Table 2. SW characteristics for ISPs interconnection agreements network

\begin{tabular}{|l|l|l|l|l|l|l|l|l|l|l|l|}
\hline & $\begin{array}{l}\text { Nb. of } \\
\text { ISPs }\end{array}$ & $\begin{array}{l}\text { Nb. of } \\
\text { agree-ments }\end{array}$ & Dia. & $\begin{array}{l}\text { Av. } \\
\text { degree }\end{array}$ & $\begin{array}{l}\text { Av. } \\
\text { L }\end{array}$ & $\begin{array}{l}\text { Av. } \\
\mathrm{L}_{R}\end{array}$ & L/L & C & $\mathrm{C}_{\mathrm{R}}$ & $\mathrm{C} / \mathrm{C}_{\mathrm{R}}$ & $\mathrm{SW}$ \\
\hline $2005-06$ & 10351 & 23712 & 4 & 4.148 & 3.643 & 6,498 & 0,560 & 0.887 & 0,000400 & 2213,22 & 3947,64 \\
\hline $2005-07$ & 11476 & 24756 & 4 & 3.930 & 3.695 & 6,830 & 0,541 & 0.822 & 0,000342 & 2400,11 & 4435,41 \\
\hline $2005-08$ & 11205 & 24606 & 5 & 3.989 & 3.683 & 6,739 & 0,546 & 0.840 & 0,000356 & 2359,32 & 4316,63 \\
\hline $2005-09$ & 10915 & 24239 & 5 & 4.016 & 3.662 & 6,687 & 0,547 & 0.834 & 0,000367 & 2266,50 & 4138,76 \\
\hline $2005-10$ & 10833 & 24469 & 5 & 4.048 & 3.662 & 6,644 & 0,551 & 0.863 & 0,000373 & 2309,29 & 4189,56 \\
\hline $2005-11$ & 10938 & 24848 & 4 & 4.113 & 3.659 & 6,576 & 0,556 & 0.866 & 0,000376 & 2302,80 & 4137,82 \\
\hline $2005-12$ & 11100 & 24653 & 5 & 4.028 & 3.671 & 6,685 & 0,549 & 0.857 & 0,000362 & 2361,43 & 4299,87 \\
\hline $2006-01$ & 10947 & 23535 & 5 & 3.908 & 3.671 & 6,823 & 0,538 & 0.847 & 0,000357 & 2372,38 & 4409,00 \\
\hline $2006-02$ & 11282 & 23882 & 5 & 3.892 & 3.685 & 6,866 & 0,536 & 0.803 & 0,000345 & 2327,50 & 4336,62 \\
\hline $2006-03$ & 10873 & 22833 & 4 & 3.857 & 3.672 & 6,885 & 0,533 & 0.855 & 0,000354 & 2410,04 & 4518,69 \\
\hline $2006-04$ & 10598 & 24357 & 5 & 4.170 & 3.643 & 6,490 & 0,561 & 0.868 & 0,000393 & 2205,80 & 3929,40 \\
\hline $2006-05$ & 10756 & 23448 & 5 & 3.978 & 3.658 & 6,723 & 0,544 & 0.855 & 0,000369 & 2311,59 & 4248,06 \\
\hline $2006-06$ & 10866 & 23958 & 5 & 4.037 & 3.655 & 6,659 & 0,548 & 0.850 & 0,000371 & 2287,65 & 4167,68 \\
\hline $2006-07$ & 11757 & 24243 & 5 & 4.113 & 3.647 & 6,627 & 0,550 & 0.844 & 0,000349 & 2412,36 & 4383,64 \\
\hline
\end{tabular}

Table 3. SW characteristics for ISPs interconnection agreements network

\begin{tabular}{|c|c|c|c|c|c|c|c|c|c|}
\hline & \multicolumn{3}{|c|}{ TOP 100} & \multicolumn{3}{|c|}{ TIER 1} & \multicolumn{3}{|c|}{ TIER 2} \\
\hline & 2005-06 & 2006-01 & 2006-07 & $2005-06$ & 2006-01 & 2006-07 & 2005-06 & 2006-01 & 2006-07 \\
\hline $\mathrm{Nb}$. of ISPs & 100 & 100 & 100 & 91 & 88 & 87 & 99 & 101 & 96 \\
\hline $\begin{array}{ll}\mathrm{Nb} . & \text { of } \\
\text { agreements } & \end{array}$ & 1964 & 1836 & 1944 & 968 & 798 & 970 & 1742 & 1680 & 1694 \\
\hline Dia. & 4 & 5 & 5 & 4 & 3 & 4 & 4 & 5 & 5 \\
\hline Av. degree & 19,52 & 18,24 & 19,54 & 10,637 & 9,068 & 11,149 & 17,596 & 16,634 & 17,646 \\
\hline Av. L & 1,974 & 2,054 & 2,054 & 2,037 & 2,055 & 2,025 & 2,029 & 2,11 & 2,048 \\
\hline Av. $L_{R}$ & 1,549 & 1,586 & 1,549 & 1,907 & 2,030 & 1,852 & 1,602 & 1,641 & 1,590 \\
\hline $\mathrm{L} / \mathrm{L}_{\mathrm{R}}$ & 1,273 & 1,295 & 1,325 & 1,067 & 1,011 & 1,093 & 1,266 & 1,285 & 1,287 \\
\hline $\mathrm{C}$ & 0,586 & 0,523 & 0,558 & 0,841 & 0,869 & 0,624 & 0,495 & 0,441 & 0,475 \\
\hline $\mathrm{C}_{\mathrm{R}}$ & 0,197 & 0,184 & 0,197 & 0,118 & 0,104 & 0,129 & 0,179 & 0,166 & 0,185 \\
\hline $\mathrm{C} / \mathrm{C}_{\mathrm{R}}$ & 2,972 & 2,838 & 2,827 & 7,115 & 8,337 & 4,813 & 2,756 & 2,651 & 2,557 \\
\hline SW & 2,333 & 2,191 & 2,132 & 6,664 & 8,239 & 4,402 & 2,177 & 2,062 & 1,985 \\
\hline
\end{tabular}


(a) Regular
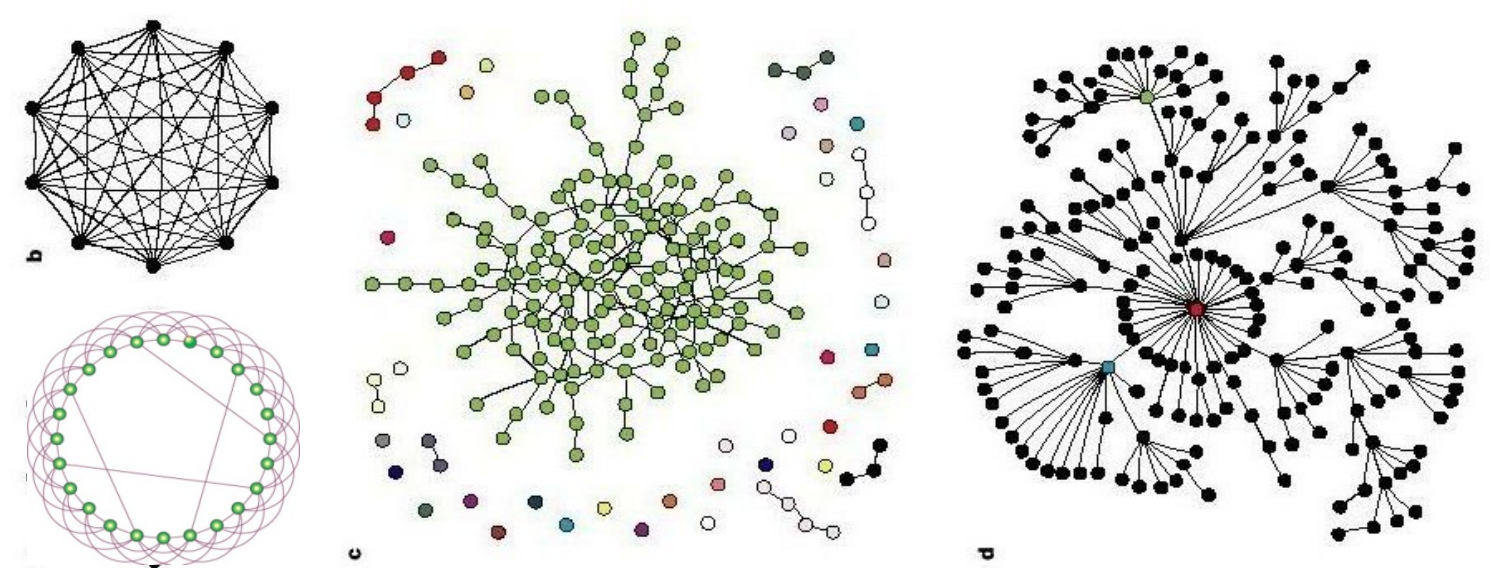

(c) Small world

(b) Random

(d) Scale free

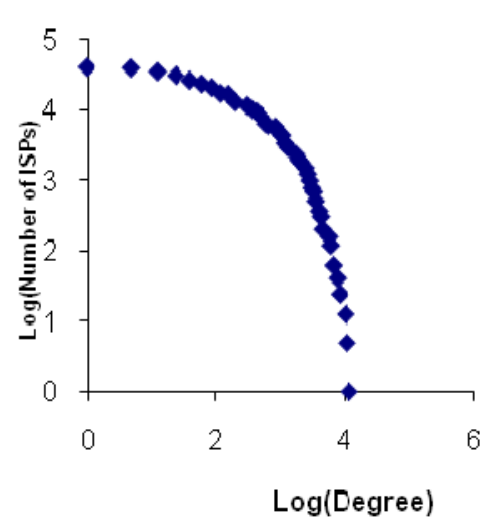

(a) Top 100

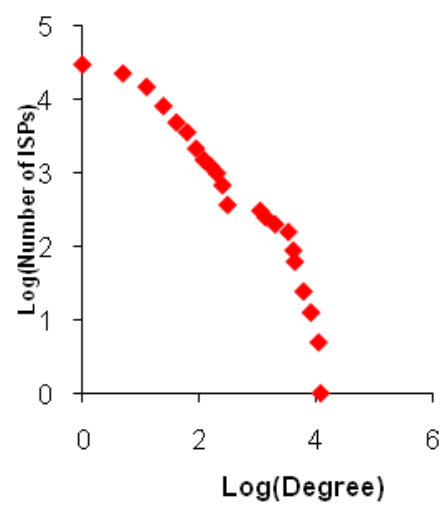

(b) Tier1

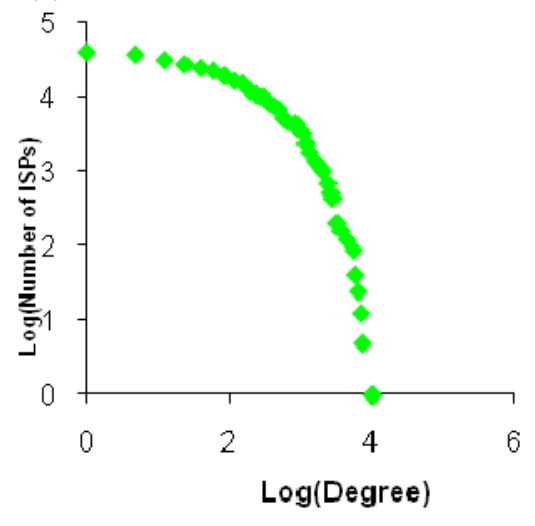

(c) Tier2

Figure 2. The log-log of the degree distribution 